\title{
Sclerosing Extramedullary Hematopoietic Tumor Mimicking Intra-abdominal Sarcoma
}

\author{
Serap Karaarslan · Nalan Nese ${ }^{1}$ Guray Oncel ${ }^{2} \cdot$ Nazan Ozsan $^{3} \cdot$ Taner Akalin $^{3}$ \\ Hasan Kaplan ${ }^{4} \cdot$ Filiz Buyukkececi ${ }^{5}$. Mine Hekimgil ${ }^{3}$ \\ Department of Pathology, Sifa University Faculty of Medicine, Izmir; 'Department of Pathology, Celal Bayar University Faculty of Medicine, Izmir; \\ ${ }^{2}$ Department of Radiology, Sifa University Faculty of Medicine, Izmir; ${ }^{3}$ Department of Pathology, Ege University Faculty of Medicine, Izmir; \\ ${ }^{4}$ Department of General Surgery, Sifa University Faculty of Medicine, Izmir; ${ }^{5}$ Department of Hematology, Kent Hospital, Izmir, Turkey
}

Sclerosing extramedullary hematopoietic tumor (SEMHT) is a rare tumor that occurs in patients with chronic myeloproliferative disorders (CMPDs). The tumor is classified in the chronic idiopathic myelofibrosis (MF) subgroup, and cases have been reported at various localizations since $1980 .{ }^{1-4}$ Such tumors are usually seen in the abdominal organs, retroperitoneum, and mesenteric region. ${ }^{3}$ The clinical, radiological, and morphological features may complicate differentiation from sarcoma, carcinoma, and lymphoma. It is sometimes also difficult to differentiate between a SEMHT and extramedullary hematopoiesis (EMH). These two lesions have similar clinical features, but EMH is morphologically more cellular. To aid in the differentiation, SEMHT has a more solid mass appearance with dense fibrosis and atypical megakaryocytes. ${ }^{3}$

$\mathrm{EMH}$ is the presence of hematopoietic tissue in locations other than the bone marrow. The basic mechanism is bone marrow dysfunction and decreased production of hematopoietic cells, followed by production of bone marrow cells in other organs and tissues. EMH is seen in many disorders such as sickle cell anemia, hemoglobinopathies, thalassemia, hereditary spherocytosis, and $\mathrm{MF}^{5} \mathrm{EMH}$ is most commonly observed in the liver and spleen and is rarely found in the peritoneum, lymph nodes, kidneys, thymus, central nervous system, retroperitoneum, myocardium, uter-

\section{Corresponding Author}

Serap Karaarslan, MD

Department of Pathology, Sifa University Faculty of Medicine, Sanayi Caddesi No. 7 ,

Bornova, Izmir 35100, Turkey

Tel: +90-232-343-4445, Fax: +90-232-343-5656

E-mail: serapkaraarslan@gmail.com

Received: January 19, 2015 Revised: April 20, 2015

Accepted: April 22, 2015 us, pleura, paraspinal region, or intestines. ${ }^{6-9}$

\section{CASE REPORT}

A 57-year-old female patient was examined for the chief complaint of fatigue. She was diagnosed with severe anemia, and a palpable intra-abdominal mass was identified on physical examination. Radiological examinations revealed hepatosplenomegaly and hypodense soft tissue lesions measuring $15 \times 6 \mathrm{~cm}$ in size along the medial liver contour at the liver portal hilus level and $9.8 \times 6 \mathrm{~cm}$ in size along the right iliac vascular structures in the right lower quadrant (Fig. 1), as well as several enlarged lymph nodes in the paraaortic and paracaval regions (the largest measured $5 \mathrm{~cm}$ and was located in the right paracaval region). Intraabdominal free fluid deposition was noted. Radiologically, the soft tissue masses observed along the medial liver contour $(15 x$ $6 \mathrm{~cm}$ ) invaded the portal vein, and the characteristics of those along the right iliac vascular structures in the right lower quadrant $(\sim 9.8 \times 6 \mathrm{~cm})$ were reported to be consistent with infiltrative sarcomatous lesion or lymphoma (diffuse infiltrative type), and pathologic evaluation was recommended. Whole blood analysis revealed a mildly increased neutrophil count $(14,500 / \mu \mathrm{L})$. Other blood analysis results were as follows: platelets $299 \times 10^{3} / \mu \mathrm{L}$, erythrocytes $3.91 \times 10^{6} / \mu \mathrm{L}$, hemoglobin $10.6 \mathrm{~g} / \mathrm{dL}$ (reference range, 10.80 to $14.90 \mathrm{~g} / \mathrm{dL}$ ), and hematocrit $34.4 \%$ (reference range, $35.60 \%$ to $45.40 \%$ ).

The patient was referred for general surgery, and the right lower quadrant mass was removed with a preliminary diagnosis of intra-abdominal malignant tumor. Macroscopically, the soft tissue mass was solid, off-white in color, and $9.5 \times 7 \times 3 \mathrm{~cm}$ in size. 
Microscopically, there were thick fibrotic bands, areas with more prominent collagen bands, mixed inflammatory cell infiltration including eosinophils, and occasional foci with individual or grouped enlarged cells with large cytoplasm and pleomorphic

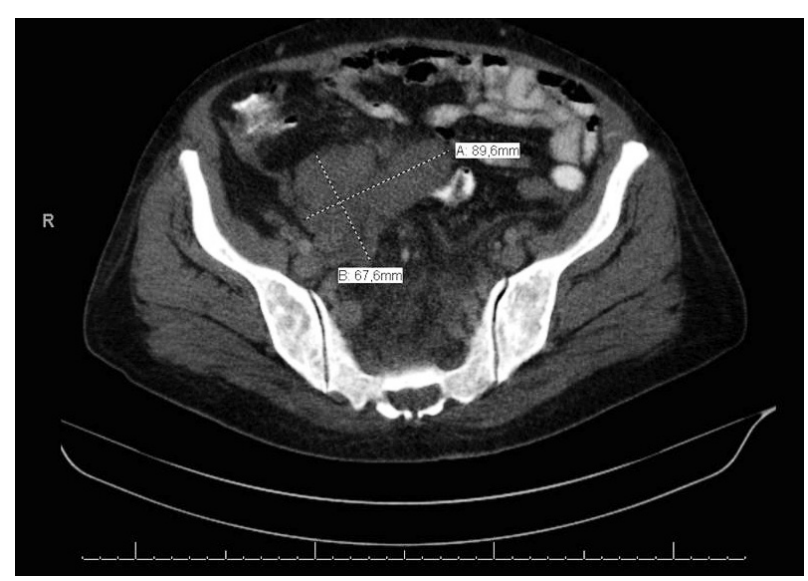

Fig. 1. A soft tissue lesion approximately $9.8 \times 6 \mathrm{~cm}$ in size with a lobulated contour along the right iliac vascular structures of the right lower quadrant. nucleoli in the background. Lymphoid follicles with prominent germinal centers were observed mostly in the periphery of the mass, and some contained the large cells described above (Fig. 2A, B). No mitosis or necrosis was found. Increased collagen was seen in the background on Masson's trichrome stain (Fig. 2C). Immunohistochemistry revealed suspicious large cells that were negative for CD34, DKA, S-100, CD31, desmin, vimentin, CD117, CD10, CD68, CD30, mast cell tryptase, CD1a, CD45, CD30, CD15, CD3, CD20, CD21, CD23, anaplastic lymphoma kinase (ALK), pancytokeratin, and epithelial membrane antigen. The Ki-67 proliferation index was very low at about $2 \%$. The background lymphoid follicle structures became more evident with CD3 and CD20 staining. Additional stains were then applied, and the large cells were positive for CD61 (Fig. $3 \mathrm{~A}$ ), indicating dysplastic megakaryocytes. Some myeloperoxidase-positive cells with mononuclear or polynuclear morphology were seen, indicating granulocytic series. Glycophorin staining revealed some precursor cells belonging to the erythroid series (Fig. 3B). We concluded that all these characteristics were re-
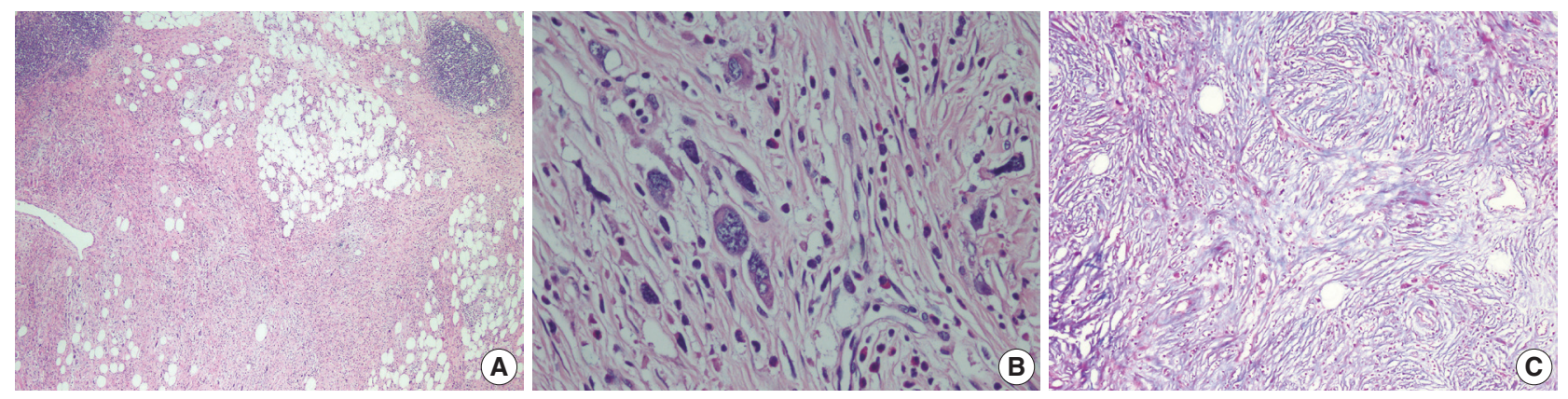

Fig. 2. (A) Thick fibrotic bands and increased collagen tissue with occasional mixed inflammatory cell infiltration including eosinophils and foci of individual or grouped cells showing pleomorphic nuclei and large cytoplasm. (B) Features of the large cells are seen more clearly at larger magnification. (C) Increased collagen is seen in the background (Masson's trichrome).
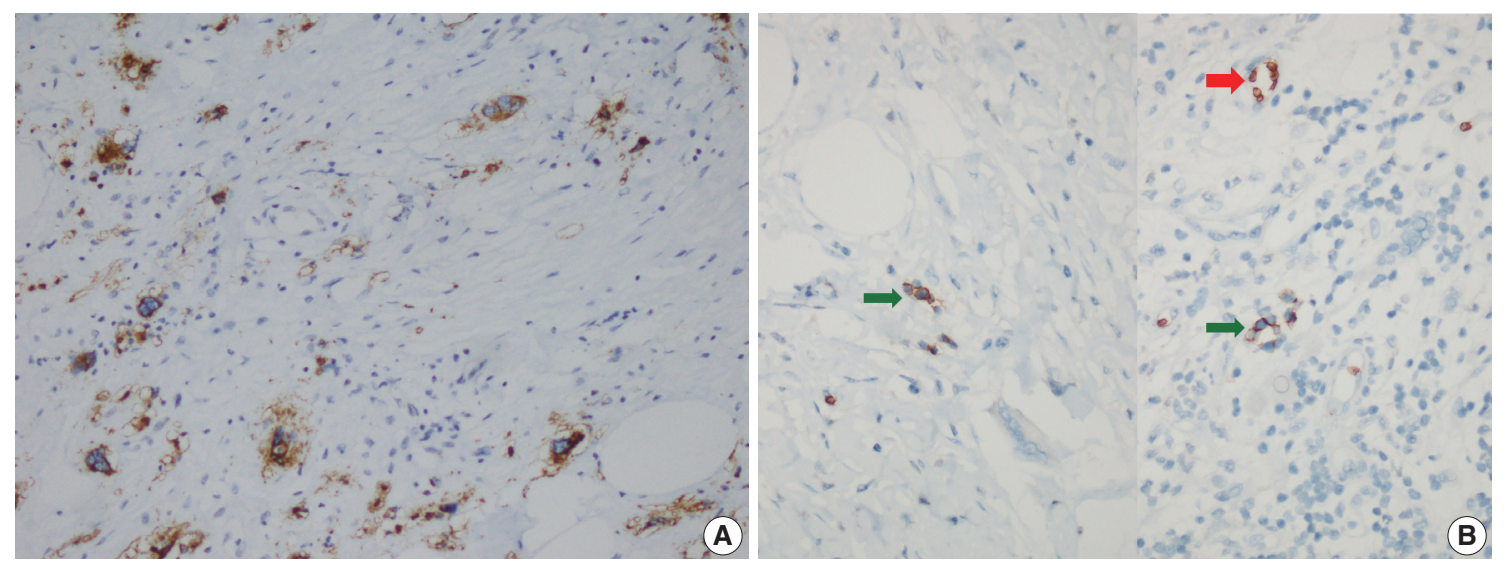

Fig. 3. (A) Large cells are CD61-positive on immunohistochemical stain, indicating that they are megakaryocytes. (B) Glycophorin positivity in precursor cells (green arrows) belonging to the erythroid lineage and surrounding erythrocytes (red arrow). 
lated to SEMHT.

Detailed investigation of the patient history revealed that she had been diagnosed with MF after a bone marrow biopsy 14 years prior. A liver biopsy had been performed at that time, and EMH was reported. The previous bone marrow biopsy evaluation found hypercellular bone marrow $(90 \%)$ with a prominent increase in occasional clustering of megakaryocytes. The bone marrow showed thick reticulin fibers with reticulin staining and collagen formation according to Masson's trichrome staining. The diagnosis of MF was made, and the patient had been followed for 14 years without treatment.

We diagnosed the retroperitoneal soft tissue mass that was highly suspicious for malignancy as SEMHT given the morphological and immunophenotypical results and the medical history. The patient was started on Hydrea (DEVA, Istanbul, Turkey) (500 g tablet, twice daily) and Urikoliz (SANDOZ, Istanbul, Turkey) (300 mg, half tablet, three times daily) to suppress the features related to SEMHT. Hydrea treatment was terminated six months later due to development of leukopenia and anemia. No other problems have developed during follow-up thus far.

\section{DISCUSSION}

SEMHT is a rare tumor more commonly seen with chronic idiopathic MF rather than CMPDs. ${ }^{1}$ It is usually located in the abdomen, retroperitoneum, and mesenteric region. ${ }^{3}$ Our patient had multiple masses located in the abdomen. EMH generally indicates the presence of bone marrow elements in an area other than the bone marrow. It can develop as a result of bone marrow failure due to various causes in a wide range of disorders including MF. EMH is most commonly observed in the spleen and liver and is rarely seen in the peritoneum, lymph nodes, kidneys, thymus, central nervous system, intestines, retroperitoneum, myocardium, uterus, pleura, or paraspinal region. ${ }^{2-6}$ Differentiation of SEMHT from EMH is made based on several characteristics such as cellularity, tendency to form a mass, and presence of fibrosis and atypical megakaryocytes. Marked cellularity indicates EMH, while the presence of fibrosis and atypical megakaryocytes and a tendency to form a mass favors a diagnosis of SEMHT. ${ }^{3}$ This case had background fibrosis, atypical megakaryocytes, and mass formation and was therefore diagnosed as SEMHT.

The differential diagnosis of large intra-abdominal or retroperitoneal soft tissue masses varies from case to case, and a clinicopathological correlation is important. A detailed immunohistochemical (IHC) panel is used together with morphological indicators of the degree of differentiation, number of mitoses, and the presence/absence of necrosis in the pathologic evaluation of soft tissue tumors. In this case, there was no necrosis in the background and no mitosis in the large cells, which did not invade the surrounding tissues, vessels, or nerves. We performed IHC stains for all entities that should be considered in the differential diagnosis of a retroperitoneal mass such as liposarcoma, malignant peripheral nerve sheath tumor, leiomyosarcoma, gastrointestinal stromal tumor, solitary fibrous tumor, inflammatory myofibroblastic tumor, and fibrosarcoma. All were negative (large cells were negative for CD34, DKA, S-100, CD31, desmin, vimentin, CD117, CD10, ALK), and the diagnosis of SEMHT was made with the help of a detailed medical history and the positivity for CD61 and glycophorin. It is difficult to differentiate some sarcomas (especially sclerosing liposarcoma) from SEMHT without using IHC markers.

Differentiating benign proliferations of bone marrow cells from those that are malignant is important. Myeloid sarcoma, which should also be considered in the differential diagnosis, is a tumor that consists of myeloid blastic cells and can develop in various anatomical sites such as the skin, lymph nodes, small intestine, and mediastinum. ${ }^{10} \mathrm{IHC}$ analysis is usually used for the differential diagnosis with hematological malignancies (large cells are negative for CD34 and CD117). We also eliminated other lymphomas including Hodgkin lymphoma (lymphocytepoor type, appearance similar to Reed-Steinberg cells in some of the large cells) and histiocytic lymphoma through IHC investigation (large cells were negative for CD68, CD30, mast cell tryptase, CD1a, CD45, CD30, CD15, CD3, CD20, CD21, CD23, ALK, and S100).

We were able to make a correct diagnosis with detailed clinicopathological correlation although the initial histopathological and radiological findings supported a possible sarcomatous neoplasm. We emphasize the importance of a thorough history and highlight the fact that radiologic and pathologic data may be difficult to interpret when the medical history is inadequate. We presented this case to remind physicians to consider this pathology in the differential diagnosis of soft tissue masses, especially when hematological disorder is also present.

\section{Conflicts of Interest}

No potential conflict of interest relevant to this article was reported.

\section{REFERENCES}

1. Remstein ED, Kurtin PJ, Nascimento AG. Sclerosing extramedul- 
lary hematopoietic tumor in chronic myeloproliferative disorders. Am J Surg Pathol 2000; 24: 51-5.

2. Sukov WR, Remstein ED, Nascimento AG, Sethi S, Lewin M. Sclerosing extramedullary hematopoietic tumor: emphasis on diagnosis by renal biopsy. Ann Diagn Pathol 2009; 13: 127-31.

3. Kwon Y, Yu E, Huh J, Lee SK, Ro JY. Sclerosing extramedullary hematopoietic tumor involving lesser omentum and ligamentumteres in liver explant. Ann Diagn Pathol 2004; 8: 227-32.

4. Yuen HK, Mahesh L, Tse RK, Yau KC, Chan N, Lam DS. Orbital sclerosing extramedullary hematopoietic tumor. Arch Ophthalmol 2005; 123: 689-91.

5. Koch CA, Li CY, Mesa RA, Tefferi A. Nonhepatosplenic extramedullary hematopoiesis: associated diseases, pathology, clinical course, and treatment. Mayo Clin Proc 2003; 78: 1223-33.
6. Hanamornroongruang S, Neungton C, Warnnissorn M. Extramedullary hematopoiesis in the uterine cervix associated with tissue repair. Case Rep Obstet Gynecol 2013; 2013: 626130.

7. Luo Y, Zhang Y, Lou SF. Bilateral pleural effusion in a patient with an extensive extramedullary hematopoietic mass. Case Rep Hematol 2013; 2013: 857610.

8. Cui X, Peker D, Greer HO, Conner MG, Novak L. Extramedullary hematopoiesis in uterine leiomyoma associated with numerous intravascular thrombi. Case Rep Pathol 2014; 2014: 957395.

9. Ahmad K, Ansari S, Koirala R, Agarwal M, Chaudhary S. Paraspinal and presacral extramedullary hematopoiesis: a rare manifestation of polycythemia vera. Iran J Radiol 2013; 10: 164-8.

10. O'Malley DP. Benign extramedullary myeloid proliferations. Mod Pathol 2007; 20: 405-15. 